\title{
A conceptual model of the diffuse transmittance of lamellar diffraction gratings on solar cells
}

\author{
K. R. Catchpole ${ }^{a)}$ \\ School of Photovoltaic and Renewable Energy Engineering, University of New South Wales, \\ Sydney NSW 2052 Australia
}

(Received 21 May 2006; accepted 2 April 2007; published online 2 July 2007)

\begin{abstract}
Diffraction gratings are effective ways of increasing the light absorption of solar cells and the light extraction of light-emitting diodes. In this paper, we show that simplified modal analysis can be used as a conceptual model for understanding the behavior of the diffuse transmittance of lamellar diffraction gratings on infinite substrates. We use simplified modal analysis to predict the optimum values of period and height for the gratings, and achieve excellent agreement with rigorous coupled wave analysis. Furthermore, we show that for thin film solar cells with front surface gratings and flat rear reflectors, modal analysis can be used to predict the optimum parameters for maximum light trapping. (C) 2007 American Institute of Physics. [DOI: 10.1063/1.2737628]
\end{abstract}

\section{INTRODUCTION}

Light trapping is becoming increasingly important in solar cells as the devices become thinner. For cells with thicknesses that can range from a few hundred nanometers for amorphous silicon to a few microns for microcrystalline silicon, conventional types of light trapping which have feature sizes around $10 \mu \mathrm{m}$ are not suitable.

Periodic and quasiperiodic diffraction grating structures are attractive alternative methods for applying light trapping to thin solar cell structures. Morf et al. introduced the use of grating structures for solar cells, studying, in particular, blazed gratings and subwavelength antireflection gratings, and showed that very high absorptances can be obtained for silicon cell thicknesses of only a few microns. ${ }^{1,2}$ Thin film $a$-Si solar cells on rectangular $\mathrm{ZnO}$ gratings were experimentally investigated by Eisele et al. ${ }^{3}$ Subsequently, a series of studies on similar structures has been carried out, ${ }^{4-7}$ since these structures allow strong light diffraction to be achieved with a relatively simple fabrication process. In these studies, the diffraction gratings were fabricated using interference lithography. Other fabrication methods include molding ${ }^{8}$ for periodic structures and nanosphere lithography for quasiperiodic structures. ${ }^{9,10}$ Quasiperiodic diffraction gratings formed by nanosphere lithography have also been used to increase the light extraction from light-emitting diodes. ${ }^{11}$

Diffraction grating structures can be modeled numerically by a number of methods, such as rigourous coupled wave analysis (RCWA). ${ }^{12-14}$ However, there are a large number of free parameters in designing a diffraction grating, and numerical modeling alone does not offer much insight as to why a particular combination of parameters should be optimal.

It has been shown recently ${ }^{15,16}$ that the modal method ${ }^{17}$ can be used as the basis for a phenomenological interpretation that allows a deeper understanding of the behavior of highly efficient transmission gratings, where two diffraction orders propagate, and other cases including the subwave-

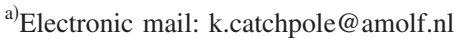

length limit, where only one diffraction order propagates. In this paper, we call this approach simplified modal analysis, and we apply the interpretation to somewhat more complicated cases, where at least three diffraction orders propagate. We show that nevertheless this simplified modal analysis can be used to provide a conceptual understanding of the diffuse transmittance of rectangular gratings suitable for solar cells. We show that the model can also be used to predict the optimum parameters for a light trapping front surface grating on a thin film solar cell with a flat rear reflector, and we confirm the results using RCWA. The approach used in this paper also provides the basis for understanding more complicated diffractive structures such as pillar-type gratings, ${ }^{18}$ which can be even more effective for light trapping and light extraction.

It is important to distinguish the diffuse transmittance used in this work from the haze parameter that has been used to characterize textured transparent conductive oxide layers designed for application in thin film $\mu \mathrm{c}-\mathrm{Si}: \mathrm{H}$ and $a$-Si solar cells. The haze parameter is the diffuse transmittance of a textured thin film measured in air, whereas the diffuse transmittance as used in this paper is the light that is transmitted diffusely into the silicon. While the diffuse transmittance into the silicon is not easily accessible experimentally, it is useful for understanding the optimal values of grating parameters. It has been pointed out recently that the haze is not a sufficient parameter to characterize the light-trapping properties of a surface texture; the angular distribution of the light contributing to the haze is also important. ${ }^{4,19}$ For a general surface texture, the diffuse transmittance into the silicon is also not a sufficient parameter to characterize light trapping because it does not contain information about the angular distribution of the light, and, in particular, the fraction of light outside the escape cone. However, for certain device structures all of the light that is diffusely transmitted lies outside the escape cone, and for such structures there is a close correlation of the maximum possible short-circuit current $J_{\text {sc }}$ with the diffuse transmittance, as described in Sec. III E. 


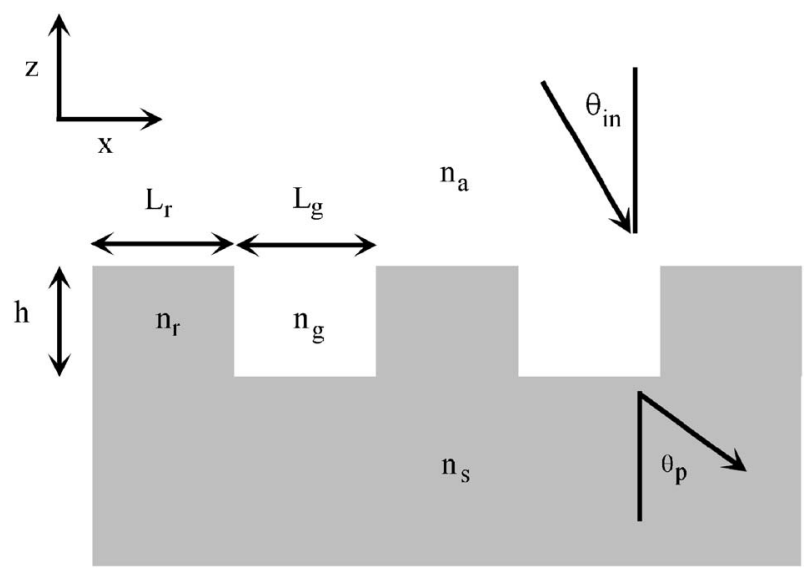

FIG. 1. Parameters for the grating under consideration.

\section{METHOD}

A diffraction grating couples incident light into a number of diffracted orders or modes. For a transmission grating on a substrate with refractive index $n_{s}$, the propagation angles of the diffracted orders are given by

$$
\sin \theta_{p}=\frac{p \lambda}{n_{s} L}, \quad p=0,1,2, \ldots,
$$

for normal incidence, where $L$ is the period of the grating. While the angles into which light is coupled are easily calculated, to find the optimum grating parameters that maximize the diffuse transmittance would generally require a complicated numerical method.

The essence of simplified modal analysis is summarized below; more details can be found in the relevant papers. ${ }^{15,16,20}$ The theory applies to rectangular (lamellar) gratings of the type shown in Fig. 1. Modal analysis divides the fields propagating in each region into different types of modes: the incident mode, modes propagating within the grating, and reflected and transmitted diffracted modes. Coupling and interference between the various modes determine the diffraction efficiency of the grating. For the grating shown in Fig. 1 the grating modes can be found analytically. Each mode is characterized by an effective refractive index $n^{\text {eff }}$ which is a solution of the equation ${ }^{20}$

$$
\begin{aligned}
f\left(n^{\mathrm{eff}}\right)= & \cos \left(k_{x r} L_{r}\right) \cos \left(k_{x g} L_{g}\right)-\frac{1}{2}\left(\tau \frac{k_{x r}}{k_{x g}}+\frac{1}{\tau} \frac{k_{x g}}{k_{x r}}\right) \\
& \times \sin \left(k_{x r} L_{r}\right) \sin \left(k_{x g} L_{g}\right)=\cos \left(k_{x} L\right),
\end{aligned}
$$

where $k_{0}$ is the wave vector of the incident wave, $k_{x}$ $=k_{0} \sin \left(\theta_{\text {in }}\right)=(2 \pi / \lambda) n_{a} \sin \left(\theta_{\text {in }}\right)$ is the $x$ component of the wave vector of the incident wave, and

$$
k_{x i}=k_{0}\left[n_{i}^{2}-\left(n^{\mathrm{eff}}\right)^{2}\right]^{1 / 2}, \quad i=r, g
$$

are the $x$ components of the wave vectors in the ridges and grooves of the grating, respectively. $\tau$ is equal to 1 for transverse-electric (TE) polarization, $n_{r}^{2} / n_{g}^{2}$ for transversemagnetic (TM) polarization, and $L=L_{r}+L_{g}$. Because of the symmetry of the problem, the field in the grating region can be separated into an $x$-dependent part $u(x)$ and a $z$-dependent part $\nu(z)$. For TE polarization, for example, only a $y$ compo- nent of the electric field is present, which can be written as

$$
E_{y}(x, z)=u(x) v(z) .
$$

The field distributions associated with each mode are given by Sheng $\mathrm{et} \mathrm{al.}{ }^{20}$ Here, we note that for normal incidence, the $x$-dependent parts of the grating modes are periodic, with the same period as the grating. One of the factors affecting the efficiency of excitation of the $m$ th grating mode by the incident mode $E_{y}^{\text {in }}$, (i.e., the fraction of power that is transferred from the incident mode to the $m$ th grating mode) is the overlap integral of the two modes, ${ }^{21}$

$$
\left\langle E_{y}^{\mathrm{in}}(x, 0), u_{m}(x)\right\rangle=\frac{\left|\int E_{y}^{\mathrm{in}}(x, 0) u_{m}(x) d x\right|^{2}}{\int\left|E_{y}^{\mathrm{in}}(x, 0)\right|^{2} d x \int\left|u_{m}(x)\right|^{2} d x} .
$$

The other factors affecting the excitation of the grating modes by the incident mode, and the excitation of diffracted modes by the grating modes, are Fresnel-like reflection and transmission coefficients. ${ }^{15,17}$ These coefficients give low reflectance and high transmittance when the $y$ components of the wave vectors of the two modes are similar. This is known as impedance matching. Thus for the air/grating interface, the efficiency of excitation of a given grating mode increases as $n^{\text {eff }}$ approaches $n_{a} \cos \theta_{\text {in }}=n_{a}$ for normally incident light. For the grating/substrate interface, the efficiency of excitation of diffracted mode $p$ by a given grating mode increases as $n^{\text {eff }}$ approaches $n_{s} \cos \theta_{p}$.

Absorption is taken into account ${ }^{20}$ in Eq. (2). However, in the calculations presented in this paper, we have neglected absorption when calculating the effective refractive indices for the various grating modes. This is a good approximation for thin silicon gratings in the long wavelength region where light trapping is important. (At short wavelengths, where silicon is strongly absorbing, it would be important to take absorption into account as the grating modes will be absorbed differently depending on their field distribution.)

\section{RESULTS AND DISCUSSION}

In this section, we first investigate how the effects of varying the period and height of silicon and $\mathrm{TiO}_{2}$ gratings can be understood with modal analysis. We then look at the relationship between diffuse transmittance and light trapping for thin silicon solar cells with front surface gratings and flat rear reflectors, and finally we apply our understanding to thin film solar cells with $\mathrm{ZnO} / \mathrm{Si}$ gratings.

\section{A. TE case}

We consider the case of TE illumination first (i.e., electric field parallel to the grooves of the grating). For a $\mathrm{Si}$ grating with $L=650 \mathrm{~nm}$ at $\lambda=1000 \mathrm{~nm}\left(n_{r}=3.58\right)$, there are three propagating grating modes with effective refractive indices $n_{0}^{\text {eff }}=3.37, n_{1}^{\text {eff }}=2.70$, and $n_{2}^{\text {eff }}=1.40$. There are also three propagating diffraction orders in the substrate. When we calculate the diffraction efficiency (i.e., the fraction of light that is transmitted into each order) as a function of height using RCWA for this case, we see an almost periodic 


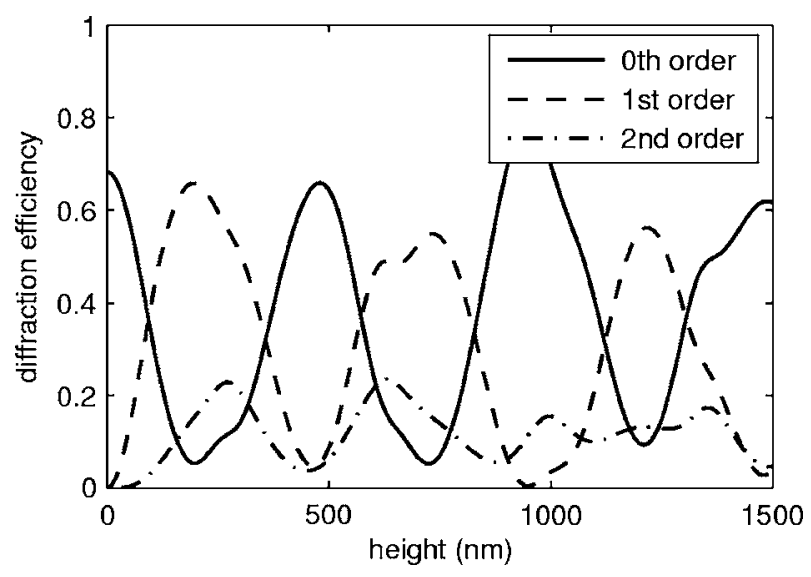

FIG. 2. The diffraction efficiency vs height $h$ for the transmitted diffracted orders for a $\mathrm{Si}$ grating on an infinite $\mathrm{Si}$ substrate with $L=650 \mathrm{~nm}$ at $L$ $=1000 \mathrm{~nm}\left(n_{r}=3.58\right)$.

oscillation of the zeroth and first diffracted orders, with much less power going into the second diffracted order (Fig. 2).

We can get a more complete picture of the grating behavior with contour plots of the diffuse transmittance as a function of wavelength and grating height. The diffuse transmittance is the fraction of light that is transmitted into the silicon into orders higher than the zeroth order, i.e., it is the total transmittance minus the zeroth order transmittance. In this section and in Secs. III B-III D, we consider the diffuse transmittance for gratings on an infinite Si substrate; in Secs. III $\mathrm{E}$ and III F, we discuss how this is related to light trapping in thin film silicon cells.

A contour plot for $L=650 \mathrm{~nm}$ calculated with RCWA is shown in Fig. 3. The major feature of the plot is a series of peaks in the diffuse transmittance at periodic values of $h$ with the peak values of $h$ decreasing with increasing wavelength. This oscillation can be explained as being due to a phase difference accumulated during propagation of the different grating modes through the grating. When the phase shift is zero essentially all the light coupled into the grating is directed into the zeroth diffracted order, while when the

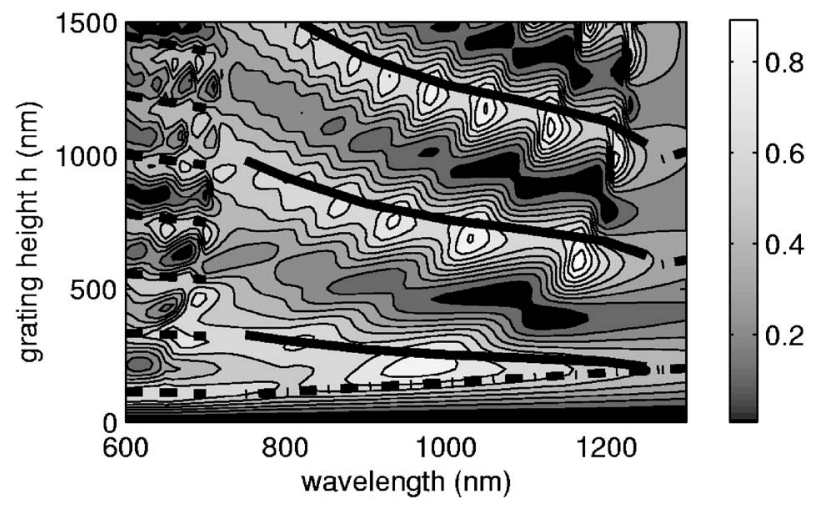

FIG. 3. Diffuse transmittance for a rectangular Si grating on an infinite Si substrate with $L=650 \mathrm{~nm}$ calculated with RCWA. The lines show the maxima predicted with modal analysis, including contributions from interference between the zeroth and second grating modes (solid lines), FabryPérot interference of the zeroth grating mode with itself (dash-dot lines), and interference between zeroth and fourth grating modes (dashed lines).
TABLE I. Overlap integrals between propagating diffracted orders $(p)$ and propagating grating modes $(m)$ for $L=650 \mathrm{~nm}$ and $\lambda=1000 \mathrm{~nm}$.

\begin{tabular}{lllllll}
\hline \hline & $n^{\text {eff }}$ & $p=-2$ & $p=-1$ & $p=0$ & $p=1$ & $p=2$ \\
\hline$m=0$ & 3.373 & 0.005 & 0.200 & 0.589 & 0.200 & 0.005 \\
$m=1$ & 2.700 & 0.108 & 0.389 & 0 & 0.389 & 0.108 \\
$m=2$ & 1.396 & 0.154 & 0.173 & 0.299 & 0.173 & 0.154 \\
\hline \hline
\end{tabular}

phase shift is $\pi$ most of the light is coupled into the first diffracted order. The peaks in the first diffracted order occur at odd integer multiples of

$$
h=\frac{\lambda}{2\left|n_{2}^{\mathrm{eff}}-n_{0}^{\mathrm{eff}}\right|} .
$$

The positions of the peaks as calculated with Eq. (6) are shown as solid black lines in Fig. 3. It can be seen that there is an excellent agreement with the results of RCWA.

To determine which modes contribute to the interference, we calculate the overlap integrals between the grating modes and the diffracted orders (Table I). For normal incidence the overlap integral between the first grating mode and the zeroth diffracted order is zero because the first grating mode is an odd function with respect to the vertical axis of symmetry of the grating. Thus for normally incident light there is no coupling to the first grating mode.

The $x$ dependence of the electric field of the grating modes for $L=650 \mathrm{~nm}$ and $\lambda=1000 \mathrm{~nm}$ is plotted in Fig. 4(a). We can see that for the zeroth grating mode most of the energy propagates in the $\mathrm{Si}$ while for the second grating mode the energy is more evenly distributed between the air and the Si. (In fact, from the intensity in each region, proportional to the square of the electric field, we find that $97 \%$ of the energy is in the $\mathrm{Si}$ for the zeroth mode, compared with $48 \%$ for the second mode.) This is the reason that the effective refractive index of the zeroth mode is high (3.37) while the effective refractive index of the second mode is relatively low (1.40). We can also use the graphs of the electric fields of the grating modes to obtain a clearer picture of the meaning of the overlap integral. In Fig. 4(b) the integrand in the numerator of Eq. (5) is plotted (shaded area), along with the $x$-dependent parts of the fields for the zeroth grating mode and first diffracted order (for which the value of the overlap integral is 0.2). The figure shows how the value of the overlap integral is increased where the fields due to both modes are high. The total value of the overlap integral is the square of the shaded area divided by the intensities of the modes taking part.

We now turn our attention to the long wavelength region of the contour plot in Fig. 3. The overlap integrals for $L$ $=650 \mathrm{~nm}$ with $\lambda=1300 \mathrm{~nm}$ are given in Table II. For $\lambda$ $=1300 \mathrm{~nm}$ there are only two propagating grating modes. The coupling between the first diffracted order and the zeroth grating mode is weaker than at $\lambda=1000 \mathrm{~nm}$, but the main difference between the two cases is that at $\lambda=1300 \mathrm{~nm}$ there is no second grating mode available to couple to the zeroth diffracted order. As at $\lambda=1000 \mathrm{~nm}$, the first grating mode does not couple with normally incident light. 

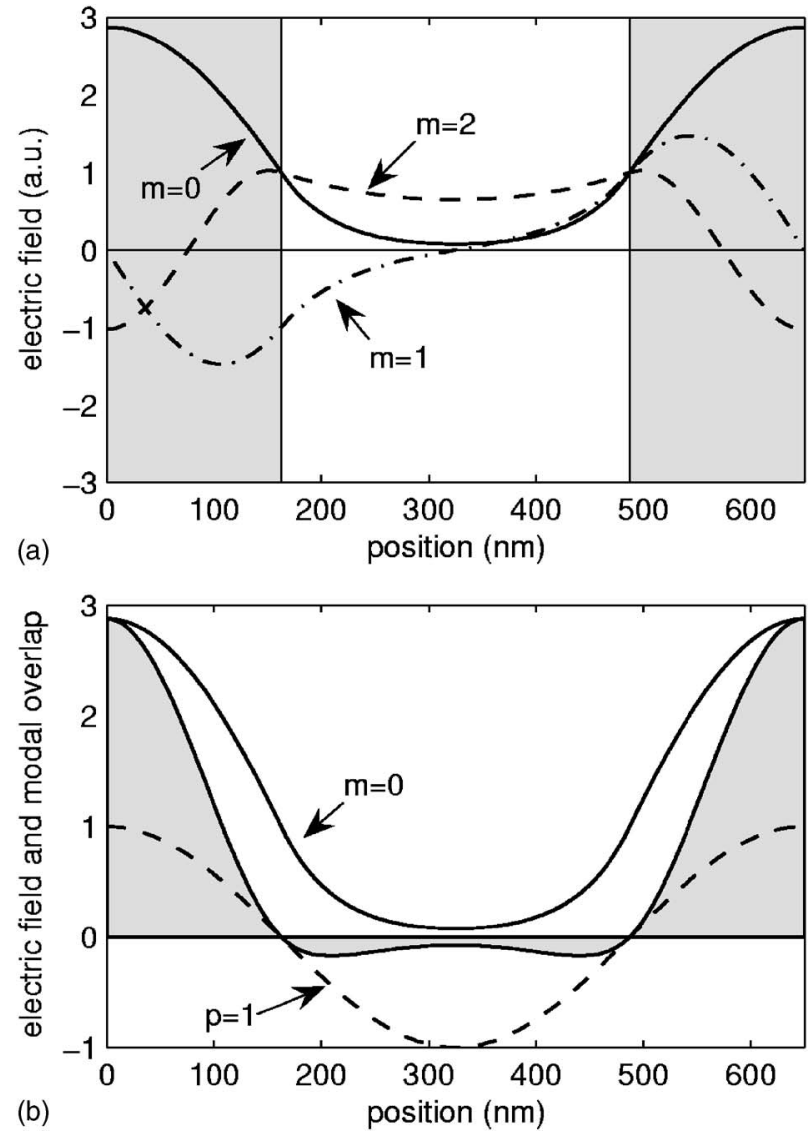

FIG. 4. (a). The $x$ dependence of the electric field of the three grating modes for $L=650 \mathrm{~nm}$ and $\lambda=1000 \mathrm{~nm}$. The gray regions are the $\mathrm{Si}$ and the white is air. (b) A schematic visualization of the meaning of the overlap integral, calculated for the overlap between the zeroth grating mode and first diffracted order for $L=650 \mathrm{~nm}$ and $\lambda=1000 \mathrm{~nm}$. The solid line shows the $x$ dependence of the zeroth grating mode while the dashed line is the $x$ dependence of the first diffracted order.

Thus interference between different grating modes does not occur for $\lambda=1300 \mathrm{~nm}$. Nevertheless we can see from Fig. 3 that there is a variation of diffuse transmittance with height for $\lambda=1300 \mathrm{~nm}$. The source of this variation is FabryPérot interference of the zeroth grating mode with itself. The Fabry-Pérot interference has maxima in the diffuse transmittance at odd integer multiples of

$$
h=\frac{\lambda}{2 n_{0}^{\mathrm{eff}}} \text {. }
$$

The maxima in diffuse transmittance according to Eq. (7) are also plotted in Fig. 3 (dash-dot lines). From the RCWA results we find that the Fabry-Pérot resonance effect becomes weaker as the grating height increases, so only the first resonance is plotted for the full wavelength range of $800-1300 \mathrm{~nm}$. The second and third resonances are plotted near $1300 \mathrm{~nm}$ only. We can see that the first maximum in

TABLE II. Overlap integrals for $L=650 \mathrm{~nm}$ with $\lambda=1300 \mathrm{~nm}$.

\begin{tabular}{lccclll}
\hline \hline & $n^{\text {eff }}$ & $p=-2$ & $p=-1$ & $p=0$ & $p=1$ & $p=2$ \\
\hline$m=0$ & 3.202 & 0.002 & 0.162 & 0.672 & 0.162 & 0.002 \\
$m=1$ & 2.103 & 0.056 & 0.444 & 0 & 0.444 & 0.056 \\
\hline \hline
\end{tabular}

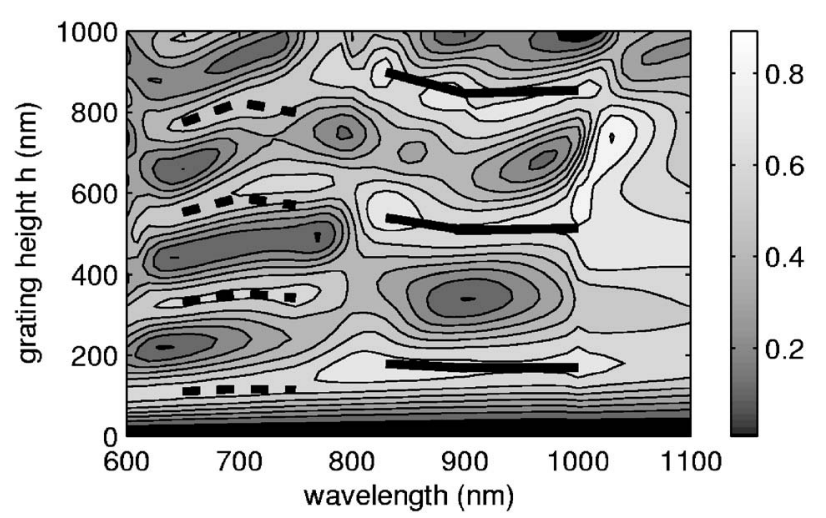

FIG. 5. Diffuse transmittance for a rectangular grating with infinite Si substrate with $L=1000 \mathrm{~nm}$. The contour plot shows the results calculated with RCWA. The lines show the maxima predicted with modal analysis, due to interference between the zeroth and fourth grating modes for $\lambda$ $=830-1000 \mathrm{~nm}$ (solid lines) and interference between the zeroth and sixth grating modes for $\lambda=650-750 \mathrm{~nm}$ (dashed lines).

diffuse transmittance as calculated by RCWA in the region where there are three propagating grating modes $(\lambda$ $=790-1250 \mathrm{~nm}$ ) is due to a combination of interference between the zeroth and second grating modes and Fabry-Pérot interference of the zeroth grating mode with itself. This leads to less variation in the optimum height with wavelength than would be the case if Fabry-Pérot resonance did not play a role.

As the wavelength decreases for a given grating period, the number of propagating grating modes increases. For example, at $\lambda=700 \mathrm{~nm}$, there are five propagating grating modes. Of these, the zeroth, second, and fourth can couple to normally incident light. The fourth grating mode has a stronger overlap with the zeroth diffracted order than the second grating mode, so interference between the zeroth and fourth grating modes dominates the height dependence. The maxima in diffuse transmittance due to interference between the zeroth and fourth grating modes are plotted in Fig. 3 for $\lambda$ in the range of $600-700 \mathrm{~nm}$. Before the onset of the fourth grating mode near $720 \mathrm{~nm}$, there is the onset of the third grating mode around $790 \mathrm{~nm}$, but this has no effect on the diffuse transmittance since it does not couple to normally incident light.

The effect of a larger period is similar to the effect of a decreased wavelength in that the number of grating modes increases. For $L=1000 \mathrm{~nm}$ in the range $\lambda=830-1000 \mathrm{~nm}$, there are five grating modes. The first and third grating modes do not couple to normally incident light, and the second grating mode has a relatively small overlap with the zeroth diffraction order of 0.09 , so the height dependence is due to interference between the zeroth and fourth grating modes (which have overlap integrals 0.50 and 0.37 , respectively), as shown in Fig. 5 (solid lines). Also shown in Fig. 5 (dotted lines) is the height dependence due to the interference of the zeroth and sixth grating modes for the wavelength range of $650-750 \mathrm{~nm}$, where there are seven propagating grating modes.

From the above examples, we can see the importance of choosing the optimum height for a given grating period. If the grating period has to be chosen larger than the optimum 

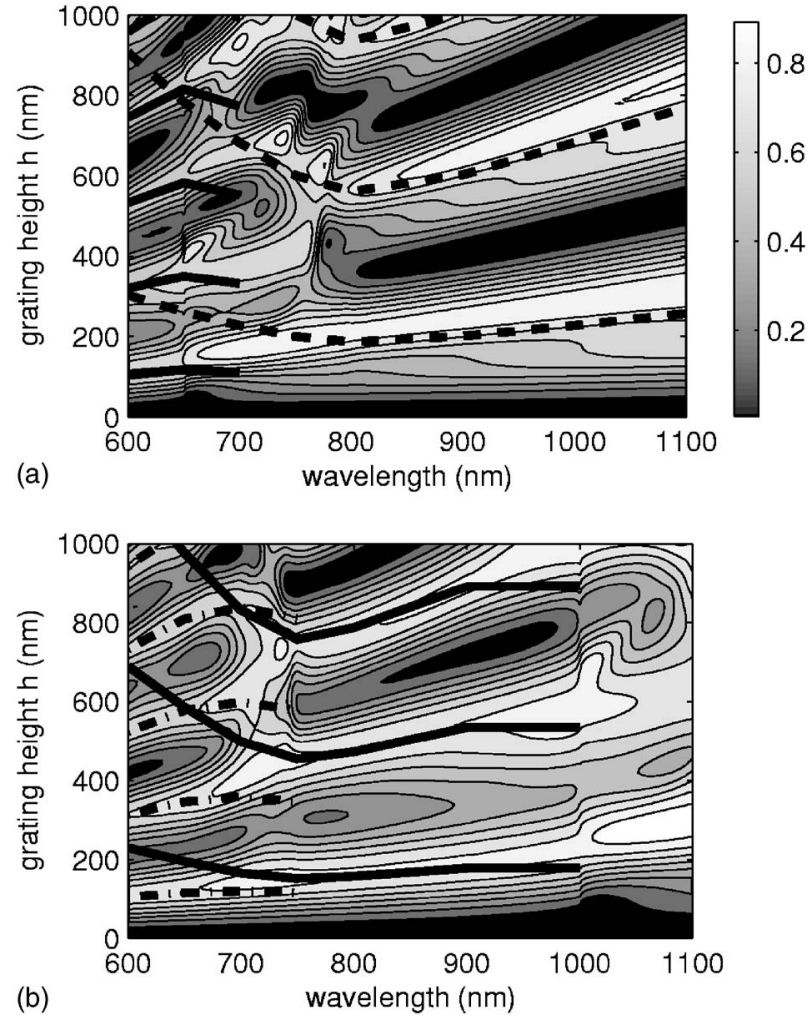

FIG. 6. Peaks in diffuse transmittance for (a) $L=650 \mathrm{~nm}$ and (b) $L$ $=1000 \mathrm{~nm}$ for the TM case for a rectangular Si grating on an infinite $\mathrm{Si}$ substrate. The dashed lines show the interference of the zeroth and second grating modes, the solid lines show the interference of the zeroth and fourth grating modes and the dash-dot lines show the interference of the zeroth and sixth grating modes. Calculation of the modal overlap allows us to predict the dominant modes in each case.

value for technological reasons, there are still sets of parameters where the diffuse transmittance is high. Modal analysis can be used to predict the optimum height for a given grating period, without the necessity of doing a full calculation using RCWA. The overlap integrals tell us which modes are important and the effective indices then allow us to calculate where the peaks in diffuse transmittance are.

\section{B. TM case}

For the TM case the diffuse transmittance can also be predicted by the interference of two grating modes, as shown in Figs. 6(a) and 6(b) and also in the next section in Fig. 7(b). We can see in Fig. 6(a) that interference between the zeroth and second grating modes gives good agreement with the diffuse transmittance peaks for $\lambda=700-1100 \mathrm{~nm}$. After the onset of the fourth grating mode at $\lambda=700 \mathrm{~nm}$, interference between the zeroth and fourth grating modes is the dominant effect. This is due to a low overlap between the second grating mode and the zeroth diffracted order, as can be seen from Table III. The trend is similar for $L=1000 \mathrm{~nm}$, as shown in Fig. 6(b). In this case, interference between the zeroth and fourth grating modes, and between the zeroth and sixth grating modes, dominates in different wavelength regions. Table III shows that in each case, the overlaps with the zeroth diffracted order are strongest for the zeroth grating mode and the highest propagating grating mode.
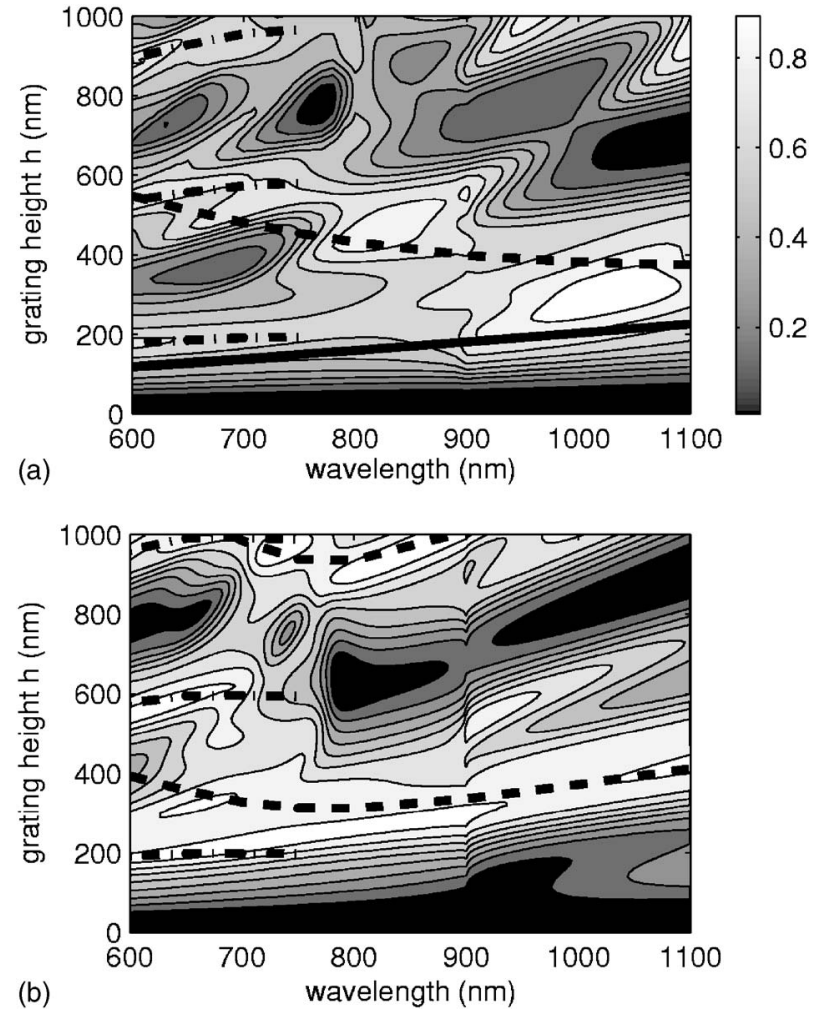

FIG. 7. Diffuse transmittance for a $\mathrm{TiO}_{2}\left(n_{s}=2.6\right)$ grating on a silicon substrate with $L=900 \mathrm{~nm}$, for (a) TE and (b) TM illuminations. The plots also show the maxima in diffuse transmittance expected due to inteference between the zeroth and second grating modes (dashed lines), between the zeroth and fourth grating modes (dash-dot lines), and Fabry-Pérot interference of the zeroth grating mode for the TE case (solid line).

\section{Criteria for high diffuse transmittance}

The design criteria for a diffraction grating for a solar cell are that diffuse transmittance should be high and that this should occur over a large wavelength range. (The diffusely transmitted light should also lie outside the escape cone; this is discussed further in Sec. III E.) From Eq. (6) we see that in order to get a high diffuse transmittance over a large wavelength range a grating height corresponding to the first peak in the diffuse transmittance (i.e., phase difference of $\pi$ rather than $3 \pi$ or $5 \pi$, etc.) should be chosen. This fixes the optimum height for a given grating period, for TE or for TM illumination. In order to choose the optimum grating period, we note that the zeroth diffraction order tends to couple most strongly with the grating modes when there are few grating modes present. Since the first grating mode does not couple

TABLE III. Overlap integrals between zeroth diffracted orders and propagating grating modes $(m)$ for TM incidence for $L=650 \mathrm{~nm}$ and $L$ $=1000 \mathrm{~nm}$, with $\lambda=600 \mathrm{~nm}$.

\begin{tabular}{llllll}
\hline \hline & \multicolumn{2}{c}{$L=650 \mathrm{~nm}$} & & \multicolumn{2}{c}{$L=1000 \mathrm{~nm}$} \\
\cline { 2 - 3 } \cline { 5 - 6 } & $n^{\text {eff }}$ & Overlap & & $n^{\text {eff }}$ & Overlap \\
\hline$m=0$ & 3.832 & 0.413 & & 3.894 & 0.411 \\
$m=2$ & 2.839 & 0.051 & & 3.510 & 0.046 \\
$m=4$ & 1.014 & 0.340 & & 2.588 & 0.020 \\
$m=6$ & $\cdots$ & $\cdots$ & & 1.031 & 0.297 \\
\hline \hline
\end{tabular}


at all to normally incident light, the most effective interference occurs between the zeroth and second grating modes.

The above considerations suggest that the largest wavelength range for diffuse transmittance will occur when there are three grating modes present (zeroth, first, and second). For $0<n^{\text {eff }}<n_{a}$ the effect of a grating mode on the interference is relatively small due to poor impedance matching with both the incident and especially the diffracted modes. Thus to get high diffuse transmittance over a large wavelength range, $n^{\text {eff }}$ for the second (or fourth, etc.) grating mode should be equal to $n_{a}$ near the longest wavelength for which light trapping is required, i.e., about $1100 \mathrm{~nm}$ for Si. To obtain high overall diffuse transmittance with a rectangular groove grating, we must have high diffuse transmittance with both TE and TM incident light. However, we find that the values of $L$ for which $n_{2}^{\text {eff }}=n_{a}=1$ are nearly identical for a $\mathrm{Si}$ /air grating for both TE and TM incidences. Thus the optimum choice for $L$ is $650 \mathrm{~nm}$ for a Si grating in air.

By knowing the periodicity of $h$, we can also estimate the height range over which we will get high diffuse transmittance. This allows the wavelength range of high diffuse transmittance to be predicted from modal theory alone, without the need for RCWA calculations.

The estimated optimum value of $h$ for TE is

$$
h_{\mathrm{opt}}^{\mathrm{TE}}(\lambda)=\frac{h_{2 \mathrm{mode}}(\lambda)+h_{\mathrm{FP}}(\lambda)}{2} \pm \frac{h_{2 \mathrm{mode}}(\lambda)-h_{\mathrm{FP}}(\lambda)}{4}
$$

where $h_{2 \text { mode }}$ is calculated from Eq. (6) and $h_{\mathrm{FP}}$ is calculated from Eq. (7). The estimated optimum value of $h$ for TM is

$$
h_{\mathrm{opt}}^{\mathrm{TM}}(\lambda)=h_{2 \text { mode }}(\lambda) \pm \frac{1}{4} h_{2 \text { mode }}(\lambda),
$$

and the optimum overall estimated value of $h$ is

$$
h_{\mathrm{opt}}(\lambda)=\frac{h_{\mathrm{opt}}^{\mathrm{TE}}(\lambda)+h_{\mathrm{opt}}^{\mathrm{TM}}(\lambda)}{2} .
$$

Equation (8) for the TE case is an average of the contributions of the two-mode interference and Fabry-Pérot interference; this was found empirically to be a good estimate of the overall effect on the optimum height when the refractive index contrast is strong, leading to high reflectivity of the air/ grating and grating substrate interfaces and hence strong Fabry-Pérot interference. For the TM case in Eq. (9), only two-mode interference occurs. The error estimates in Eqs. (8) and (9) are $\frac{1}{4}$ of the period of the variation in diffraction efficiency with height. Equation (10) is valid when the peak diffraction efficiencies for TE and TM illuminations are similar, which is the case for the diffraction gratings and illumination conditions investigated in this paper. We can obtain a wavelength independent optimum by averaging $h_{\mathrm{opt}}$ over the wavelength range where the number of grating modes present does not change. If the estimated optimum values for TE and TM are close in value, we can expect that the overall diffuse transmittance will be high.

\section{Predicting optimum parameters for gratings with different refractive indices}

We can use modal analysis to make predictions of the optimum choice of parameters for gratings without the need to perform RCWA. Although RCWA can provide more information than the simplified modal analysis used here, often modal analysis is all that is needed to predict the range where the optimum values will be found.

As an example, we look at a grating made of $\mathrm{TiO}_{2}$ (on a silicon substrate) that will be the "equivalent" of the optimal case of $L=650 \mathrm{~nm}$ for a silicon grating. As for the silicon grating, we expect that the optimal light diffusion will occur when $n_{2}^{\text {eff }}=1$ occurs near $\lambda=1100 \mathrm{~nm}$. For a $\mathrm{TiO}_{2}$ grating this is when $L=900 \mathrm{~nm}$, for both TE and TM illuminations. The results of RCWA for this grating are plotted in Fig. 7, along with the maxima in diffuse transmittance expected from Eqs. (6) and (7). Simplified modal analysis thus predicts a broad optimum in diffuse transmittance in the height range indicated on the figure. Thus a simple calculation allows us to predict an optimal value of $L$ and optimal range for $h$ for a given grating refractive index. Using Eqs. (8)-(10) we can predict that the optimum height for TE is $295 \pm 50 \mathrm{~nm}$ and the optimum height for TM is $358 \pm 90 \mathrm{~nm}$, over the wavelength range of 800-1100 nm. This leads to an overall optimum height of $327 \pm 70 \mathrm{~nm}$, which is in very good agreement with the optimum height as calculated by RCWA of $300 \pm 5 \mathrm{~nm}$. We can see that simplified modal analysis can be used to choose the optimum period for a given grating material and can also predict the optimum height of a given grating structure.

\section{E. Diffuse transmittance and light trapping}

For thin film structures with gratings that have sufficiently low periods, all of the light that is diffusely transmitted into the silicon lies outside the escape cone. This occurs for structures which satisfy the criterion established by Eisele et al. ${ }^{3}$ of $L<p \lambda$ for all $\lambda$ of interest, where $p$ is the diffracted order. Here, we show that this results in a close correlation of the maximum possible $J_{\text {sc }}$ with the diffuse transmittance for a thin film structure with a front surface grating and flat rear reflector. The period range examined here also includes the overall optimum period, since at larger periods, some of the diffused light lies within the escape cone, leading to lower absorptance. Figure 8(a) shows the diffuse transmittance $T_{d}$ versus grating height $h$ for a silicon grating on an infinite silicon substrate with $L=650 \mathrm{~nm}$ at $\lambda=950 \mathrm{~nm}$, along with the maximum possible $J_{\text {sc }}$ of a $3 \mu \mathrm{m}$ thick Si device with the same front surface grating and a flat Ag reflector. The maximum possible $J_{\text {sc }}$ was calculated by integrating the absorptance calculated with RCWA over the AM1.5G spectrum, i.e., the internal quantum efficiency of the device was assumed to be equal to unity. For the wavelength range of $850-1100 \mathrm{~nm}$, we can see that there is a close correlation between the diffuse transmittance and $J_{\mathrm{sc}}$ as a function of grating height, particularly in the region of the first peak, and that there is excellent agreement on the overall optimum grating height at $h=200 \mathrm{~nm}$. There is a shift in the position of the second peak between the $J_{\mathrm{sc}}$ and diffuse transmittance 

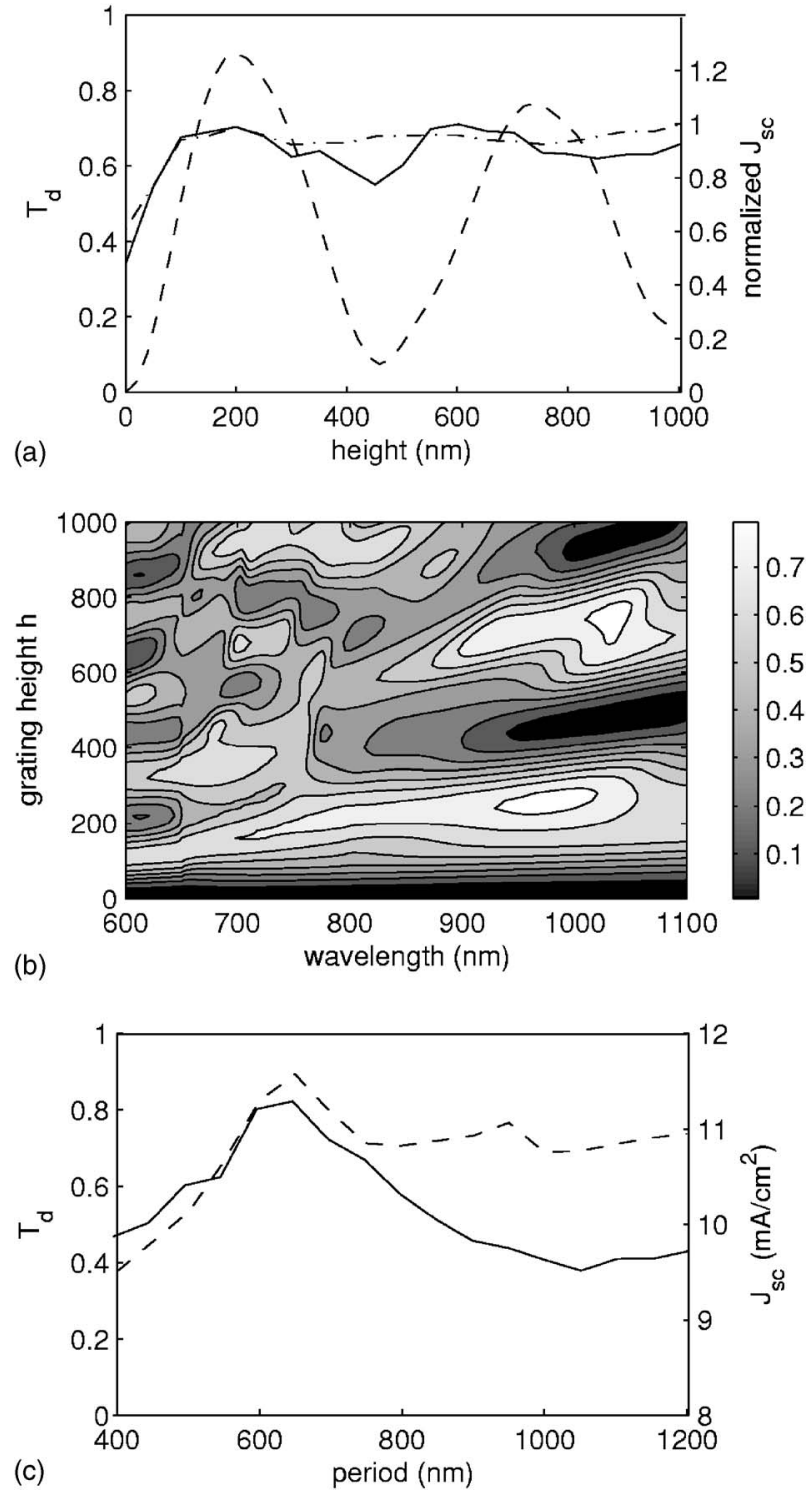

FIG. 8. (a) $T_{d}$ vs $h$ for an infinite Si substrate with $\lambda=950 \mathrm{~nm}$ (dashed line), and $J_{\text {sc }}$ vs $h$ for a $3 \mu \mathrm{m} \mathrm{Si}$ active layer for $\lambda>650 \mathrm{~nm}$ (solid line) and for $\lambda>850 \mathrm{~nm}$ (dash-dot line) $(L=650 \mathrm{~nm})$. The $J_{\mathrm{sc}}$ results have been normalized to their maximum values to facilitate comparison with the $T_{d}$ results. (b) Contour plot of $T_{d}$ vs $h$ and $\lambda$ for $L=650 \mathrm{~nm}$, averaged over TE and TM illuminations. (c) $T_{d}$ vs $L$ for an infinite $S i$ substrate with $\lambda=950 \mathrm{~nm}$ (dashed line) and $J_{\text {sc }}$ for $\lambda>650 \mathrm{~nm}$ for a $3 \mu \mathrm{m} \mathrm{Si}$ active layer (solid line) ( $h$ $=200 \mathrm{~nm}$ ).

results; this is because the optimum value for the second peak is more sensitive to wavelength than the value for the first peak, so the overall position of the second peak depends on the relative contribution of each wavelength to $J_{\text {sc }}$. The variation of the heights of the secondary peak also leads to a much weaker dependence on $h$ for the wavelength range of $650-1100 \mathrm{~nm}$ than for the wavelength range of $850-1100 \mathrm{~nm}$, for values of $h$ above $200 \mathrm{~nm}$. We can see the reason for this in Fig. 8(b), which shows a contour plot of diffuse transmittance versus $h$ and $\lambda$, averaged over TE and TM incidences. In the wavelength range of $600-800 \mathrm{~nm}$ the positions of the second, third peaks, etc., change rapidly as new grating modes appear. However, the position of the first peak is still relatively independent of wavelength, and this value determines the overall optimum for maximum $J_{\mathrm{sc}}$. The variation of diffuse transmittance with grating period at $\lambda$ $=950 \mathrm{~nm}$, together with $J_{\mathrm{sc}}$ for the thin film structure calculated over the wavelength range of $650-1100 \mathrm{~nm}$, is shown in Fig. 8(c). Again we see good agreement between the optimum period and the overall optimum, because the optimum period is relatively independent of wavelength. In this case, a secondary peak that appears in the diffuse transmittance at $L=950 \mathrm{~nm}$ does not appear in the $J_{\mathrm{sc}}$ result because its position varies strongly with wavelength, and it is much weaker for shorter wavelengths.

Thus, in the region near the overall optimum period, the diffuse transmittance is a good predictor of light trapping for a front grating structure with a flat rear reflector, noting that the relative contribution of different wavelengths will affect the results. The grating parameters for the overall maximum value of $J_{\mathrm{sc}}$ can be predicted. Secondary maxima can also be predicted where they are relatively independent of wavelength over a given wavelength range. In some cases the secondary maxima are strongly dependent on wavelength. In these cases the secondary maxima in values for $J_{\mathrm{sc}}$ can only be estimated from modal analysis, because the relative contribution of each wavelength to the final $J_{\text {sc }}$ is not known from modal analysis alone.

For the device structures studied here with periods near the optimum value, diffuse transmittance provides a good measure of light trapping. As the period increases, the correlation between diffuse transmittance and light trapping will decrease as more diffracted orders come to lie within the escape cone. Thus for a more general device structure, including rectangular gratings where the periods are significantly higher than optimal for technological reasons, it would be necessary to take into account the angular distribution of the flux to fully characterize the light trapping. However, diffuse transmittance into the silicon will remain an important consideration in all cases. (This is different from the case of haze, where there may be zero haze and yet still significant light trapping. ${ }^{22}$ )

\section{F. Behavior of $\mathrm{ZnO} / \mathrm{Si}$ gratings}

In this section we consider the case of a $\mathrm{ZnO} / \mathrm{Si}$ grating, with light incident from the $\mathrm{ZnO}$. Grating structures formed from these materials have been the subject of recent experimental and numerical studies, because the structure can be fabricated relatively simply by deposition of silicon on a $\mathrm{ZnO}$ grating/thin layer structure which in turn lies on a glass superstrate. ${ }^{4-7}$ The experimental structures also have a rear silicon grating because the silicon is deposited conformally on the $\mathrm{ZnO}$. Here, since we are focussing on single grating structures, we restrict ourselves to comparison with structures with a front surface $\mathrm{ZnO} / \mathrm{Si}$ grating and a flat rear reflector, as studied numerically by Haase and Stiebig. ${ }^{7}$ Figure 9 shows the diffuse transmittance versus grating period and height for $\mathrm{ZnO} / \mathrm{Si}$ gratings on an infinite silicon substrate, under TE and TM illuminations at a wavelength of $950 \mathrm{~nm}$, in the middle of the light-trapping range. The optimum height values predicted by modal analysis are also shown, and as before there is very good agreement between the modal analysis and RCWA results. For good impedance 

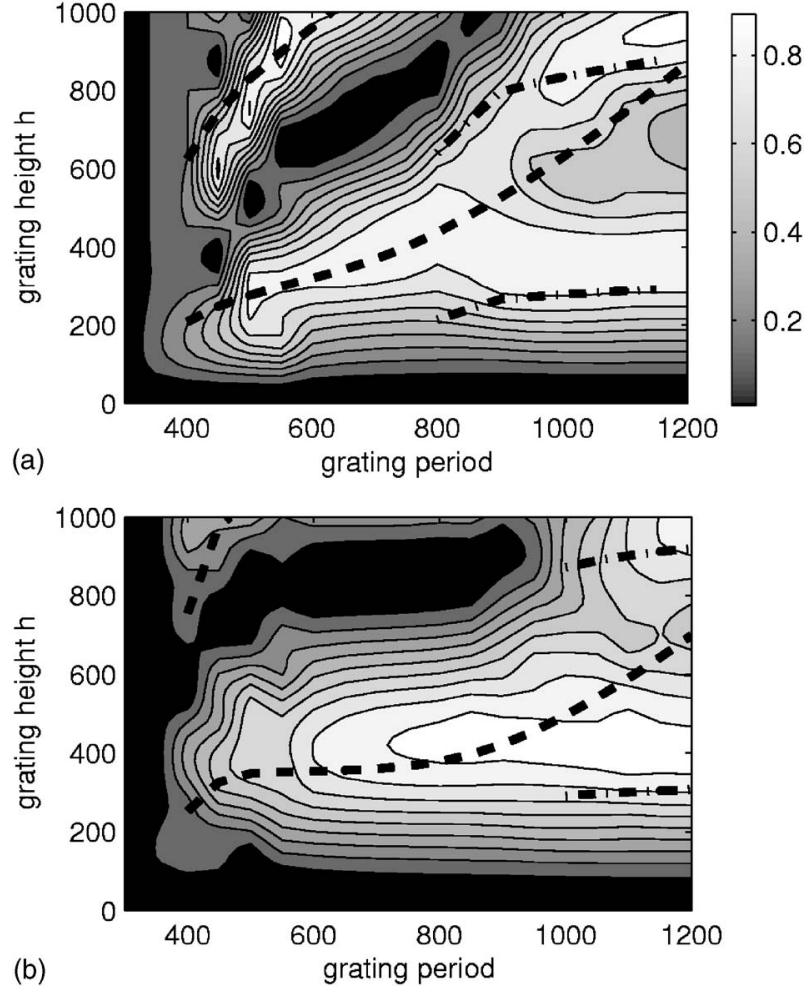

FIG. 9. (a) TE and (b) TM plots of $T_{d}$ vs $L$ and $h$ for $\mathrm{Si} / \mathrm{ZnO}$ grating on an infinite $\mathrm{Si}$ substrate at $\lambda=950 \mathrm{~nm}$. The dashed lines are due to interference between the zeroth and second grating modes, and the dash-dot lines are due to interference between the zeroth and fourth grating modes.

matching, modal analysis predicts that the optimum period should occur where $n_{2}^{\text {eff }} \cong n_{a}=2.0$, which occurs at $L$ $=750 \mathrm{~nm}$ for both TE and TM illuminations for $\lambda$ $=1100 \mathrm{~nm}$, the maximum wavelength for which light trapping is required. This value is in very good agreement with the period that was found by Haase and Stiebig ${ }^{7}$ to give maximum current for the thin film structure of $L=700 \mathrm{~nm}$. Thus also in this case modal analysis can predict parameters for maximizing both the diffuse transmittance and the maximum possible short-circuit current. For $L=750 \mathrm{~nm}$, the optimum height predicted by modal analysis at $\lambda=950 \mathrm{~nm}$, averaged over TE and TM illuminations is $383 \mathrm{~nm}$. Haase and Stiebig did not calculate the optimum height for a front grating structure, but it is interesting to note that the optimum period and height predicted by modal analysis for a front grating structure are also very close to the optimum period and height predicted for a structure with both a front grating and a rear reflection grating ${ }^{7}$ of $L=700 \mathrm{~nm}$ and $h$ $=300-350 \mathrm{~nm}$. Although an investigation of the behavior of dual grating structures is beyond the scope of this paper, this result suggests that near the optimum parameters for the front surface grating, the performance of the front surface grating may be the dominant factor in the light-trapping be- havior of a dual grating cell. This is supported by the fact that near the optimum parameters for the front surface grating, single grating and dual grating structures had similar predicted $J_{\mathrm{sc}}$ values under long wavelength illumination. ${ }^{7}$

\section{CONCLUSIONS}

We have shown that simplified modal analysis can be used to understand the diffuse transmittance behavior of onedimensional (1D) lamellar gratings for solar cells, and that it can be used to predict the optimum values for 1D gratings, without the need to undertake rigorous numerical modeling. For thin film solar cells with a front surface grating and flat rear reflector, this allows us to predict the optimum period and height for maximum light trapping. The approach used here also provides the basis for understanding more complicated diffractive structures that may be even more effective for light trapping and light extraction, such as pillar-type gratings. ${ }^{18}$

\section{ACKNOWLEDGMENTS}

One of the authors (K.R.C.) acknowledges the support of an Australian Research Council fellowship, and also the support of the Centre of Excellence for Advanced Silicon Photovoltaics and Photonics, supported by the Australian Research Council.

${ }^{1}$ R. H. Morf, H. Kiess, and C. Heine, in Diffractive Optics for Industrial and Commercial Applications, edited by J. Turunen and F. Wyrowski (Akademie, Berlin, 1997).

${ }^{2}$ C. Heine and R. H. Morf, Appl. Opt. 34, 2476 (1995).

${ }^{3}$ C. Eisele, C. E. Nebel, and M. Stu0tzmann, J. Appl. Phys. 89, 7722 (2001).

${ }^{4}$ H. Stiebig, N. Senoussaoui, C. Zahren, C. Haase, and J. Muller, Prog. Photovoltaics 14, 13 (2006).

${ }^{5}$ N. Senoussaoui, M. Krause, J. Muller, E. Bunte, I. Brammer, and H. Stiebig, Thin Solid Films 451-452, 397 (2004).

${ }^{6}$ H. Stiebig, C. Haase, C. Zahren, B. Rech, and N. Senoussaoui, J. NonCryst. Solids 352, 1949 (2006).

${ }^{7}$ C. Haase and H. Stiebig, Prog. Photovoltaics 14, 629 (2006).

${ }^{8}$ O. Azzaroni, P. L. Schilardi, R. C. Salvarezza, J. Manuel-Herrero, C. Zaldo, and L. Vazquez, Appl. Phys. A: Mater. Sci. Process. 81, 1113 (2005).

${ }^{9}$ H. W. Deckman and J. H. Dunsmuir, J. Vac. Sci. Technol. B 1, 1109 (1983).

${ }^{10}$ J. C. Hulteen and R. P. Vanduyne, J. Vac. Sci. Technol. A 13, 1553 (1995).

${ }^{11}$ R. Windisch et al., IEEE Trans. Electron Devices 47, 1492 (2000).

${ }^{12}$ M. G. Moharam and T. K. Gaylord, J. Opt. Soc. Am. 72, 1385 (1982).

${ }^{13}$ M. G. Moharam, E. B. Grann, D. A. Pommet, and T. K. Gaylord, J. Opt. Soc. Am. A 12, 1068 (1995).

${ }^{14}$ Grating Solver Development Company, 2004.

${ }^{15}$ A. V. Tishchenko, Opt. Quantum Electron. 37, 309 (2005).

${ }^{16}$ T. Clausnitzer, T. Kampfe, E. B. Kley, A. Tunnermann, U. Peschel, A. V. Tishchenko, and O. Parriaux, Opt. Express 13, 10448 (2005).

${ }^{17}$ L. C. Botten, M. S. Craig, R. C. McPhedran, J. L. Adams, and J. R. Andrewartha, Opt. Acta 28, 413 (1981).

${ }^{18}$ K. R. Catchpole and M. A. Green, J. Appl. Phys. 101, 063105 (2007).

${ }^{19}$ J. Krc, F. Smole, and M. Topic, Prog. Photovoltaics 11, 429 (2003).

${ }^{20}$ P. Sheng, R. S. Stepleman, and P. N. Sanda, Phys. Rev. B 26, 2907 (1982).

${ }^{21}$ A. Yariv, IEEE J. Quantum Electron. QE9, 919 (1973).

${ }^{22}$ V. Terrazzoni-Daudrix et al., Prog. Photovoltaics 14, 485 (2006). 